This is the peer reviewed version of the following article:

Geographic variations in stage at diagnosis and survival for colorectal cancer in Australia: A systematic review

\author{
European journal of cancer care \\ First published: 05 May 2019 \\ which has been published in final form at \\ https://onlinelibrary.wiley.com/doi/abs/10.1111/ecc.13072
}

This article may be used for non-commercial purposes in accordance with Wiley Terms and Conditions for Self-Archiving." 


\title{
Geographic variations in stage at diagnosis and survival for colorectal cancer in Australia: A systematic review
}

\author{
Fiona Crawford-Williams ${ }^{1}$ (D) $\mid$ Sonja March $^{1,2}$ | Belinda C. Goodwin ${ }^{1}$ (D) | Michael \\ J. Ireland $^{1,2}$ | Suzanne K. Chambers ${ }^{1,3,4,5,6}$ (D) | Joanne F. Aitken ${ }^{1,3,7}$ | Jeff Dunn ${ }^{1,3,5,6,8}$
}

${ }^{1}$ Institute for Resilient Regions, University of Southern Queensland, Springfield Central, Queensland, Australia

${ }^{2}$ School of Psychology, University of Southern Queensland, Springfield Central, Queensland, Australia

${ }^{3}$ Cancer Research Centre, Cancer Council Queensland, Fortitude Valley, Queensland, Australia

${ }^{4}$ Menzies Health Institute Queensland, Griffith University, Southport, Queensland, Australia

${ }^{5}$ Health and Wellness Institute, Edith Cowan University, Joondalup, Western Australia, Australia

${ }^{6}$ Faculty of Health, University of Technology Sydney, Sydney, New South Wales, Australia

${ }^{7}$ School of Public Health and Social Work, Queensland University of Technology, Brisbane, Queensland, Australia

${ }^{8}$ School of Social Science, The University of Queensland, Brisbane, Queensland, Australia

\section{Correspondence}

Fiona Crawford-Williams, Institute for Resilient Regions, University of Southern Queensland, USQ Springfield Campus, 37 Sinnathamby Blvd, Springfield Central, QLD 4300, Australia.

Email: Fiona.crawford-williams@usq.edu.au

\begin{abstract}
Introduction: Australia has one of the highest incidence rates of colorectal cancer (CRC) in the world. Residents in rural areas of Australia experience disadvantage in health care and outcomes. This review investigates whether patients with CRC in rural areas demonstrate poorer survival and more advanced stages of disease at diagnosis.

Methods: Systematic review of peer-reviewed articles and grey literature. Studies were included if they provided data on survival or stage of disease at diagnosis across multiple geographical locations; focused on CRC patients; and were conducted in Australia.

Results: Twenty-six articles met inclusion criteria. Twenty-three studies examined survival, while five studies investigated stage at diagnosis. The evidence suggests that non-metropolitan patients are less likely to survive CRC for five years compared to patients living in metropolitan areas, yet there was limited evidence to suggest geographical disparity in stage of diagnosis.

Conclusions: While five-year survival disparities are apparent, these patterns appear to vary as a function of specific region and health jurisdiction, cancer type and year/s of data collection. Future research should examine current data using consistent and robust methods of reporting survival and classifying geographical location. The impact of population-level screening programmes on survival and stage at diagnosis also needs to be thoroughly explored.
\end{abstract}

\section{KEYWORDS}

colorectal cancer, cancer screening, cancer survival, population health, systematic review, rural health, health disparities

\section{INTRODUCTION}

Australia has one of the highest incidence rates of colorectal cancer (CRC) in the world, with cases expected to continue increasing (Australian Institute of Health \& Welfare, 2017b; Ferlay et al., 2013). It is estimated that one in 13 Australians will receive a CRC diagnosis during their lifetime. CRC is the second most common cancer type among both men and women in Australia, following prostate and breast cancer (Australian Institute of Health \& Welfare, 2017b), and responsible for the second highest cancer-related burden of disease (Australian Institute of Health \& Welfare, 2017a). As the primary cause of death for approximately 4,120 Australians in 2014 (9\% of total cancer deaths), CRC represents a substantial and increasing burden to the population's health and the nation's health resources. 
It is estimated that on average, Australians diagnosed with CRC have a $68 \%$ chance of surviving for five years or more (Australian Institute of Health \& Welfare, 2017b). Unfortunately, residents of regional, rural and remote areas of Australia experience greater disadvantage in health care and outcomes (Duckett, 2016). In terms of cancer care, this is observed in lower involvement in preventative approaches, reduced access to timely and up-to-date treatments, limited psychosocial support and reduced access to specialist care (Australian Institute of Health \& Welfare, 2016; Dunn et al., 2013; Lejeune et al., 2010; Veitch, Crossland, Steeghs, Ho, \& Hanks, 2008). These disadvantages may contribute to reduced CRC survival rates for Australians living outside metropolitan centres. An important contributor to reduced survival rates in non-metropolitan areas is that individuals residing in these areas may be diagnosed at a later stage of disease (Baade, Dasgupta, Aitken, \& Turrell, 2011b). For example, evidence has indicated that patients in rural areas are more likely to be diagnosed with advanced cancers, including breast and lung (Heathcote \& Armstrong, 2007; Jong et al., 2004; NguyenPham, Leung, \& McLaughlin, 2014).

A recent systematic review reported evidence of geographic variations in CRC management and outcomes (Ireland et al., 2017). However, the evidence relating to survival was not conclusive, and evidence regarding stage at diagnosis was insufficient. These findings underscored the need for a more in-depth examination of geographical disparities in survival and stage at diagnosis utilising clinical and government reports as well as peer-reviewed literature. Thus, the current review involves a comprehensive and focused literature search, containing potentially informative "grey" literature (e.g. government reports). The aim was to systematically retrieve and synthesise all available evidence relating to geographic variations in CRC stage at diagnosis and survival rates in Australia.

\section{2 | METHODS}

The review protocol was registered with PROSPERO (https://www. crd.york.ac.uk/PROSPERO/display_record.asp?ID=CRD4201604 2666) and was conducted and reported following the PRISMA statement (Liberati et al., 2009). Two clinical questions guided the review:

1. For individuals diagnosed with $C R C$, do those who reside in non-metropolitan areas have poorer survival rates than those living in metropolitan areas of Australia?

2. For individuals diagnosed with CRC, do those living in non-metropolitan areas have a more advanced stage of cancer at diagnosis compared with those living in metropolitan areas of Australia?

\section{1 | Search strategy}

The literature search followed a two-stage process. First, peer-reviewed publications were searched utilising CINAHL, Medline, Psyclnfo, Embase and Informit databases. The search covered all articles in these databases from January 1990 to January 2019. Search terms were based on keywords, subject headings and $\mathrm{MeSH}$ terms relating to "colorectal cancer" or "bowel cancer," and "survival" or "mortality" or "cancer staging" or "spread." Terms relating to geographic variation included "geography" or "metropolitan" or "urban" or "rural" or "remote" or "regional." Full syntax for the electronic search is available in Appendix A.

An additional search of grey literature was conducted through searching key government websites, Australian cancer organisations such as state Cancer Councils, Internet search engines (Google) and bibliographies of included studies.

\subsection{Eligibility criteria}

Studies were included if data reported were from Australia; the sample included patients with CRC or there was a CRC-specific subgroup; data were presented on metropolitan versus non-metropolitan comparisons; data reported on outcome measures pertaining to survival or mortality (relative risk, hazard ratio or standardised mortality etc.) and/or stage at diagnosis. Studies were excluded if the study was qualitative, or if the study was not available in English. Review articles, theses, conference proceedings, editorials and commentaries were also excluded.

\section{3 | Study selection and data extraction}

After removing duplicates, titles and abstracts were independently screened by two reviewers to determine relevance according to the selection criteria. Full-text versions of each article deemed to be relevant were then retrieved and screened. Reviewer discrepancies at either stage were discussed and resolved within the project team. Data extracted from each included study were study population, diagnosis period, rural/urban definition, measure of survival or stage at diagnosis and key findings. It was determined that a meta-analysis could not be undertaken due to the inconsistent definitions of rurality and lack of standardisation in data presentation.

\subsection{Risk of bias assessment}

Quality assessments were conducted using a tool developed for research in breast cancer, based on the Newcastle-Ottawa Scale (Wells et al., 2000; Youl et al., 2016). Individual studies were rated on a scale of 0 (high risk of bias), 1 (intermediate risk of bias) and 2 (low risk of bias) according to nine criteria and then categorised into "high" (14-18), "moderate" (9-13) or "low" (<9) overall quality.

\section{3 | RESULTS}

Database searching identified 457 records in total. After removal of duplicates, 390 records were screened by title and abstract. Of those, 95 full-text articles were deemed relevant to the current research questions and were assessed for eligibility. Following full-text 


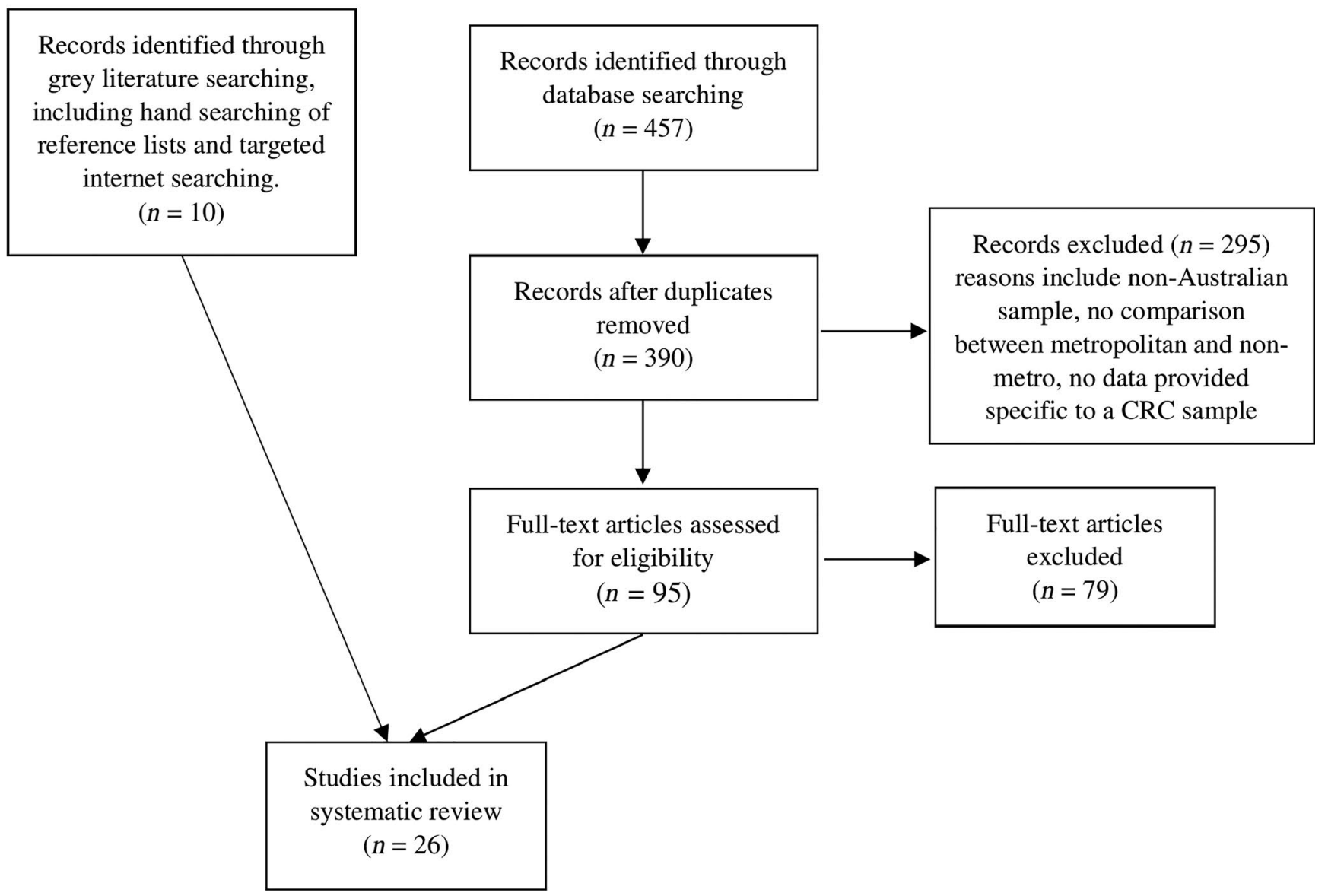

FIGURE 1 Flow diagram of included studies

assessment, 16 peer-reviewed articles were included in the final review. Grey literature searching identified 10 additional reports to be included in the review, thus the total number of included studies was 26 (see Figure 1).

\section{1 | Study characteristics}

Of the 26 studies included, 23 reported on a comparison in survival rates between CRC patients in metropolitan versus non-metropolitan areas, and 5 studies provided information on disparities in stage at diagnosis, with two studies investigating both. Tables 1 and 2 provide details of the included studies, such as author, design, population, outcome, key findings and quality rating. Where studies included findings from several time periods (e.g. 1980-1986 and 2005-2010), we chose to report findings from the most recent period. This allowed us to make interpretations based on the most current findings.

\section{2 | Study quality}

Overall, the evidence reviewed was generally of high quality. The majority of studies ( $N=17,65 \%)$ received a high-quality rating, while the rest were of moderate quality ( $N=9,35 \%)$, and no studies were classified as low quality. The substantive meaning of the results did not appear to vary according to study quality. For the studies that examined survival disparities, 15 were high quality and of these, seven showed significant geographical disparities. Eight studies were deemed moderate quality with four of these reporting significant disparities. For studies examining differences in stage at diagnosis, four studies were considered to be high quality and one (from the grey literature) was moderate. Again this did not appear to have any bearing on the significance of findings.

\section{3 | Geographical classification}

Studies included in this review used various methods to classify geographical location. A number of studies adopted a simple classification of metropolitan versus non-metropolitan, whereas others utilised standardised classification systems such as the Australian Standard Geographic Classification (ASGC) Remoteness Areas and the Accessibility/Remoteness Index of Australia (ARIA). The ASGC and ARIA classification systems both allocate local government areas to one of five categories. These categories are as follows: major city (or highly accessible); inner regional (or accessible); outer regional (or moderately accessible); remote; and very remote. However, due to smaller numbers in regional locations, many studies combine areas into three (metropolitan, regional, remote) or four categories (metropolitan, inner regional, outer regional and remote/very remote). 
TAB LE 1 Characteristics of included studies (geographic variations in CRC survival)

\begin{tabular}{|c|c|c|c|c|c|}
\hline Study & Design and data source & $\begin{array}{l}\text { Diagnosis } \\
\text { Period }\end{array}$ & Population & Rural/urban definition & Outcome(s) \\
\hline \multicolumn{6}{|l|}{ Peer-reviewed studies } \\
\hline $\begin{array}{l}\text { Baade, Dasgupta, } \\
\text { Aitken, and } \\
\text { Turrell (2011a) }\end{array}$ & $\begin{array}{l}\text { Retrospective population- } \\
\text { wide registry study (QLD) }\end{array}$ & $1996-2006$ & $\begin{array}{l}\text { All patients aged 20-79 } \\
\text { diagnosed with inva- } \\
\text { sive rectal cancer in } \\
\text { QLD }(N=6,848)\end{array}$ & $\begin{array}{l}\text { Distance and travel } \\
\text { time to radiotherapy } \\
\text { facilities calculated } \\
\text { using GIS applications }\end{array}$ & $\begin{array}{l}\text { Cause-specific } 5 \text { year relative } \\
\text { survival rates }(\%)\end{array}$ \\
\hline $\begin{array}{l}\text { Baade, Dasgupta, } \\
\text { Aitken, and } \\
\text { Turrell (2013) }\end{array}$ & $\begin{array}{l}\text { Retrospective population- } \\
\text { wide registry study (QLD) }\end{array}$ & 1996-2007 & $\begin{array}{l}\text { Patients aged } \\
20-84 \text { years diag- } \\
\text { nosed with invasive } \\
\text { stage I-IV CRC } \\
(N=22,727)\end{array}$ & ARIA + (4 categories) & $\begin{array}{l}\text { Cause-specific } 5 \text { year relative } \\
\text { survival rates }(\%)\end{array}$ \\
\hline $\begin{array}{l}\text { Beckmann et al. } \\
(2016)^{*}\end{array}$ & $\begin{array}{l}\text { Retrospective population- } \\
\text { wide registry study (SA) }\end{array}$ & 2003-2008 & $\begin{array}{l}\text { All cases of CRC } \\
\text { aged } 50-79 \text { years } \\
(N=4,641)\end{array}$ & ARIA (5 categories) & $\begin{array}{l}\text { Cause-specific } 5 \text { year relative } \\
\text { survival rates }(\%)\end{array}$ \\
\hline Chen et al. (2015) & $\begin{array}{l}\text { Retrospective popula- } \\
\text { tion-wide registry study } \\
\text { (NSW) }\end{array}$ & $2000-2008$ & $\begin{array}{l}\text { Patients diagnosed } \\
\text { with any of } 5 \text { cancers } \\
\text { ( } N=39,333 \mathrm{CRC} \\
\text { patients) }\end{array}$ & ARIA+ (4 categories) & $\begin{array}{l}\text { Cause-specific } 5 \text { year relative } \\
\text { survival rates }(\%)\end{array}$ \\
\hline $\begin{array}{l}\text { Coory et al. } \\
(2013)\end{array}$ & $\begin{array}{l}\text { Retrospective population- } \\
\text { wide mortality analysis } \\
\text { using ABS data }\end{array}$ & 2001- 2010 & $\begin{array}{l}\text { Australia wide CRC } \\
\text { deaths }\end{array}$ & $\begin{array}{l}\text { ASGC: ( } 2 \text { catego- } \\
\text { ries-metropolitan or } \\
\text { "regional and remote") }\end{array}$ & $\begin{array}{l}\text { Excess cause-specific deaths and } \\
\text { age-standardised mortality ratios } \\
\text { (SMRs) }\end{array}$ \\
\hline $\begin{array}{l}\text { Cramb et al. } \\
(2012)\end{array}$ & $\begin{array}{l}\text { Retrospective population- } \\
\text { wide registry study (QLD) }\end{array}$ & 1996-2007 & $\begin{array}{l}\text { Patients < } 90 \text { years in } \\
\text { Queensland diagnosed } \\
\text { with CRC }(N=26,390)\end{array}$ & $\begin{array}{l}\text { Distance to treatment: } \\
<2 \mathrm{hr}, 2-6 \mathrm{hr} \text {, and } 6+\mathrm{hr}\end{array}$ & $\begin{array}{l}\text { Relative excess risk of cancer } \\
\text { death (RER) within } 5 \text { years of } \\
\text { diagnosis }\end{array}$ \\
\hline
\end{tabular}

\begin{tabular}{|c|c|c|c|c|c|}
\hline Hall et al. (2005) & $\begin{array}{l}\text { Retrospective population- } \\
\text { wide data linkage study } \\
\text { (WA) }\end{array}$ & 1991-2001 & $\begin{array}{l}\text { WA residents with di- } \\
\text { agnosis of invasive pri- } \\
\text { mary CRC }(N=10,217)\end{array}$ & ARIA (5 categories) & Five-year all-cause mortality \\
\hline $\begin{array}{l}\text { Hocking et al. } \\
(2014)\end{array}$ & $\begin{array}{l}\text { Retrospective population- } \\
\text { wide data linkage study } \\
\text { (SA Clinical Registry for } \\
\text { Metastatic CRC) }\end{array}$ & 2006-2012 & $\begin{array}{l}\text { SA patients with meta- } \\
\text { static CRC }(N=2,289)\end{array}$ & $\begin{array}{l}\text { Postcodes within state } \\
\text { capital Adelaide were } \\
\text { "city" cohort; remain- } \\
\text { ing postcodes were } \\
\text { "rural" cohort }\end{array}$ & Median overall survival (months) \\
\hline
\end{tabular}


Key findings
Statistical inference of

differences
Study quality Evidence summary
Survival rates across different road dis-

tances: $<50 \mathrm{~km}=64.5 \%$; $50-99 \mathrm{~km}=60.9 \%$;

$100-199 \mathrm{~km}=59.6 \% ; 200-399 \mathrm{~km}=58.0 \%$;

$>400 \mathrm{~km}=57.4 \%$.

Adjusted hazard ratio $(95 \% \mathrm{Cl}):<50 \mathrm{~km}=1.00$;

$50-99 \mathrm{~km}=1.03(0.87-1.22) ; 100-199 \mathrm{~km}=1.16$

(1.00-1.34); $200-399 \mathrm{~km}=1.30$ (1.15-1.47);

$>400 \mathrm{~km}=1.25(1.07-1.47)$.

Survival rates across different travel times:

$0-1 \mathrm{hr}=64.3 \% ; 1-2 \mathrm{hr}=59.4 \% ; 2-4 \mathrm{hr}=58.3 \%$;

$4-6 \mathrm{hr}=58.6 \% ;>6 \mathrm{hr}=55.1 \%$.

Adjusted hazard ratio $(95 \% \mathrm{Cl}): 0-1 \mathrm{hr}=1.00$;

$1-2 \mathrm{hr}=1.07(0.93-1.23) ; 2-4 \mathrm{hr}=1.28(1.13-1.45)$;

$4-6 \mathrm{hr}=1.24(1.08-1.44) ;>6 \mathrm{hr}=1.22(0.85-1.76)$.

Survival rates by region: major city $=68.1 \%$, inner regional $=65.9 \%$, outer regional $=61.6 \%$, remote (includes very remote) $=59.6 \%$.

Adjusted odds ratio $(95 \% \mathrm{Cl})$ : major city $=1.00$; inner regional $=0.98(0.90,1.06)$; outer regional $=1.15$ $(1.05,1.25)$; remote $=1.24(1.07,1.42)$.

Survival rates across ARIA regions: inner urban $=69 \%$, outer urban $=71 \%$, rural $=68 \%$, remote $=64 \%$, very remote $=59 \%$

Hazard ratio $(95 \% \mathrm{Cl})$ : urban $=1.00$; outer

urban $=0.95(0.79-1.16)$; rural $=0.98(0.82-1.17)$;

remote $=1.12(0.90-1.39)$.

\section{Chi-square tests for distance}

model: $p<0.001$

Chi-square test for travel time

model: $p<0.001$
High (16)

After adjusting for age, sex and stage at diagnosis, patients who lived $100-199 \mathrm{~km}$, $200-399 \mathrm{~km}$ and $400 \mathrm{~km}$ or more from a radiotherapy facility were $16 \%, 30 \%$ and $25 \%$, respectively, more likely to die from rectal cancer than patients living within $50 \mathrm{~km}$ of such a facility.

Similarly, patients who travelled $2-4 \mathrm{hr}$, $4-6 \mathrm{hr}$ and $>6 \mathrm{hr}$ for treatment were $28 \%$, $24 \%$ and $22 \%$, respectively, more likely to die from rectal cancer than patients travelling up to $1 \mathrm{hr}$.
After adjusting for age, sex and stage at diagnosis, patients living in outer regional and remote areas were significantly more likely to die from colorectal cancer; however, patients in inner regional areas were less likely to die than metropolitan patients.

\section{Kaplan-Meier log-rank test for High (16)} ARIA: $p=0.219$

Survival rates across regions were not significantly different for all stages; however, patients residing in remote locations had significantly worse outcomes than metropolitan residents, though this was only evident for stages A-C (HR $=1.35,95 \% \mathrm{Cl}$ 1.01-1.80).

Significant differences in survival identified in adjusted regression models: Metastatic colon cancer in Outer Regional areas ( $H R=1.14,1.01-1.29)$; and localised rectal cancer in Outer Regional areas (HR $=1.68$, 1.02-2.77) and for regionalised rectal cancer in Inner Regional $(H R=1.14,1.002-1.30)$.

For men with CRC: number of excess cancer deaths in regional and remote areas $=407(95 \% \mathrm{Cl}, 242-576)$. SMR = 1.05 (1.02-1.09).

For women with CRC: number of excess cancer deaths in regional and remote areas $=343(95 \% \mathrm{Cl}$, 197-492) compared with metro areas. $\mathrm{SMR}=1.06$ (1.02-1.10)

Survival estimates: $<2 \mathrm{hr}=64.9 \% ; 2-6 \mathrm{hr}=60.6 \%$; $>6 \mathrm{hr}=59.7 \%$.

Adjusted RER estimates: $<2 \mathrm{hr}=1.00 ; 2-6 \mathrm{hr}=0.99$

(0.91-1.06); >6 hr= 1.07 (0.97-1.16).

Of the 6,019 CRC deaths within 5 years of diagnosis in QLD, 470 (8\%) would not have occurred if there were no spatial inequalities in the non-diagnostic survival component (after adjusting for stage, age and gender)

\begin{tabular}{|c|c|c|c|}
\hline $\begin{array}{l}\text { Cox regression analysis or the likelihood of death } \\
\text { from any cause according to ARIA }(\mathrm{HR}, 95 \% \mathrm{CI}) \text { : Very } \\
\text { accessible = HR } 1.00 \text {; accessible }=0.85(0.71-1.02) ; \\
\text { mod accessible }=1.10(0.90-1.34) ; \text { remote }=1.19 \\
(0.85-1.66) \text {; very remote }=1.05(0.71-1.54)\end{array}$ & Not reported & High (17) & $\begin{array}{l}\text { Residency in rural areas or admission to a } \\
\text { rural hospital had no effect on the likelihood } \\
\text { of death. }\end{array}$ \\
\hline $\begin{array}{l}\text { Median survival: } \\
\text { city }=14.6 \text { months } \\
\text { rural }=14.9 \text { months }\end{array}$ & Log-rank test: $p=0.18$ & $\begin{array}{l}\text { Moderate } \\
\text { (13) }\end{array}$ & $\begin{array}{l}\text { No significant differences in median survival } \\
\text { were noted between metropolitan and rural } \\
\text { patients. }\end{array}$ \\
\hline
\end{tabular}
Log-rank test: $p<0.05$

High (15)

After adjusting for gender and age, where significant differences were found, major cities tended to show the best survival, whereas outer regional areas tended to show the worst for both metastatic colon and localised rectal cancer.

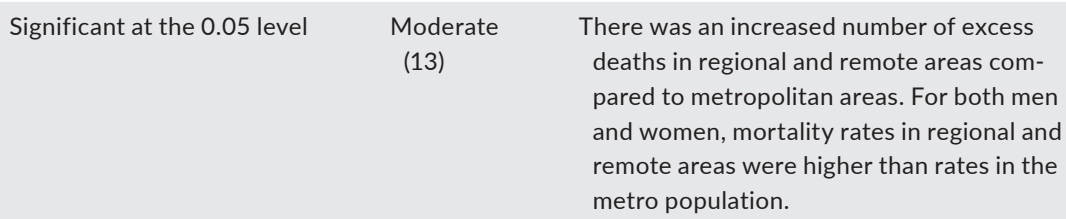
pared to metropolitan areas. For both men and women, mortality rates in regional and remote areas were higher than rates in the metro population.

$$
\text { Log-rank test: } p<0.001 \quad \text { High (17) }
$$

Risk of death was slightly lower for patients travelling 2-6 hr to treatment and higher for patients travelling $6+\mathrm{hr}$ to treatment compared to those travelling less than $2 \mathrm{hr}$ to treatment (adjusting for age, gender, stage, distance and area disadvantage). 
TABLE 1 (Continued)

\begin{tabular}{|c|c|c|c|c|c|}
\hline Study & Design and data source & $\begin{array}{l}\text { Diagnosis } \\
\text { Period }\end{array}$ & Population & Rural/urban definition & Outcome(s) \\
\hline $\begin{array}{l}\text { Jong et al. } \\
\qquad(2004)^{*}\end{array}$ & $\begin{array}{l}\text { Retrospective popula- } \\
\text { tion-wide registry study } \\
\text { (NSW) }\end{array}$ & 1992-1996 & $\begin{array}{l}\text { All patients diagnosed } \\
\text { with any cancer } \\
(N=132,516)\end{array}$ & ARIA (3 categories) & $\begin{array}{l}\text { Relative excess risk of cancer } \\
\text { death (RER) within } 5 \text { years of } \\
\text { diagnosis }\end{array}$ \\
\hline $\begin{array}{l}\text { Martin et al. } \\
(2015)\end{array}$ & $\begin{array}{l}\text { Retrospective popula- } \\
\text { tion-wide data linkage } \\
\text { and registry study (Royal } \\
\text { Perth Hospital-WA) }\end{array}$ & 2007-2011 & $\begin{array}{l}\text { Patients diagnosed with } \\
\text { any advanced cancer } \\
(N=395 \mathrm{CRC})\end{array}$ & $\begin{array}{l}\text { Residential postcode: } \\
\text { regional }(>6,211) \text { ver- } \\
\text { sus metropolitan Perth } \\
(6,000-6211)\end{array}$ & Median overall survival (months) \\
\hline $\begin{array}{l}\text { Roder et al. } \\
\text { (2015) }\end{array}$ & $\begin{array}{l}\text { Retrospective population- } \\
\text { wide registry study (SA) }\end{array}$ & $1980-2010$ & $\begin{array}{l}\text { South Australian CRC } \\
\text { patients }(N=9,098)\end{array}$ & $\begin{array}{l}\text { Postcode of resi- } \\
\text { dence: metro or } \\
\text { non-metropolitan }\end{array}$ & $\begin{array}{l}\text { Cause-specific } 5 \text { year relative } \\
\text { survival rates }(\%)\end{array}$ \\
\hline $\begin{array}{l}\text { Singla et al. } \\
\text { (2014) }\end{array}$ & $\begin{array}{l}\text { Retrospective cohort } \\
\text { study using South } \\
\text { Australian Clinical } \\
\text { Registry for Metastatic } \\
\text { Colorectal Cancer }\end{array}$ & $2006-2012$ & $\begin{array}{l}\text { Patients with metastatic } \\
\text { CRC }(N=2,001)\end{array}$ & ASGC (4 categories) & $\begin{array}{l}\text { Cause-specific } 5 \text { year relative } \\
\text { survival rates }(\%)\end{array}$ \\
\hline $\begin{array}{l}\text { Tervonen et al. } \\
\text { (2017) }\end{array}$ & $\begin{array}{l}\text { Retrospective popula- } \\
\text { tion-wide registry study } \\
\text { (NSW) }\end{array}$ & $1980-2008$ & $\begin{array}{l}\text { Patients with CRC } \\
(N=101,402)\end{array}$ & ARIA+ (3 categories) & Relative risk of cancer death \\
\hline $\begin{array}{l}\text { Wilkinson and } \\
\text { Cameron (2004) }\end{array}$ & $\begin{array}{l}\text { Retrospective population- } \\
\text { wide registry study (SA) }\end{array}$ & 1977-1993 & \multicolumn{2}{|l|}{$\begin{array}{l}\text { All cancer patients in SA } \\
\text { ( } N=82,000 \text { total-no } \\
\text { breakdown of CRC } \\
\text { cases) }\end{array}$} & $\begin{array}{l}\text { Cause-specific } 5 \text { year relative } \\
\text { survival rates }(\%)\end{array}$ \\
\hline Study & Design and data source & $\begin{array}{l}\text { Diagnosis } \\
\text { period }\end{array}$ & Population & Rural/urban definition & Outcome(s) \\
\hline \multicolumn{6}{|c|}{ Grey Literature reports } \\
\hline $\begin{array}{l}\text { Australian } \\
\text { Institute of } \\
\text { Health and } \\
\text { Welfare (2007) }\end{array}$ & $\begin{array}{l}\text { Retrospective popula- } \\
\text { tion-wide study (ABS } \\
\text { mortality database) }\end{array}$ & 2002-2004 & All CRC patients & ASGC (5 categories) & $\begin{array}{l}\text { Standardised cause-specific mor- } \\
\text { tality ratio (SMR) }\end{array}$ \\
\hline $\begin{array}{l}\text { Australian } \\
\text { Institute of } \\
\text { Health and } \\
\text { Welfare (2012) }\end{array}$ & $\begin{array}{l}\text { Retrospective population- } \\
\text { wide study (Australian } \\
\text { Cancer Database) }\end{array}$ & $2006-2010$ & All CRC patients & ASGC (4 categories) & $\begin{array}{l}\text { Cause-specific } 5 \text { year relative } \\
\text { survival rates }(\%)\end{array}$ \\
\hline $\begin{array}{l}\text { Australian } \\
\text { Institute of } \\
\text { Health and } \\
\text { Welfare } \\
\text { (2017b) }\end{array}$ & $\begin{array}{l}\text { Retrospective population- } \\
\text { wide study (Australian } \\
\text { Cancer Database) }\end{array}$ & $2010-2014$ & All CRC patients & ARIA+ (5 categories) & $\begin{array}{l}\text { Age-standardised mortality rate } \\
\text { (ASR) and rate ratio (RR) }\end{array}$ \\
\hline $\begin{array}{l}\text { Cancer Institute } \\
\text { NSW (2018) }\end{array}$ & $\begin{array}{l}\text { Retrospective popula- } \\
\text { tion-wide registry study } \\
\text { (NSW) }\end{array}$ & 2005-2009 & $\begin{array}{l}\text { All cancer } \\
\text { patients aged } \\
15-100 \text { years }\end{array}$ & ARIA (4 categories) & $\begin{array}{l}\text { Cause-specific } 5 \text { year relative } \\
\text { survival rates }(\%)\end{array}$ \\
\hline
\end{tabular}




\section{Key findings}

Five-year adjusted RER for colon cancer $(95 \% \mathrm{Cl})$ : highly accessible $=1.00$ (ref); accessible $=1.15$ (1.05-1.25); mod accessible = 1.30 (1.04-1.63); and remote $=1.01$ (0.61-1.68).

Five-year adjusted RER for rectal cancer: highly accessible = 1.00 (ref); accessible = 1.07 (0.94-1.21); mod accessible $=1.22(0.92-1.62) ;$ and remote $=2.32$ (1.38-3.89)
Statistical inference of differences

Colon: $p=0.006 \quad$ High (16)

Rectal: $p=0.02$

\section{Study quality Evidence summary}

After adjusting for stage at diagnosis, age, sex and years since diagnosis risk of death increased in accessible and moderately accessible but not remote areas for colon cancer. Increased risk of death was reported with increasing distance from highly accessible areas in rectal cancer. No significant difference in models without stage of disease as a covariate.

Median survival:

Metropolitan $=13.1$ months

Log-rank test $p=0.32$

Moderate

(11)

Regional $=16.4$ months

$\mathrm{HR}=1.13 ; 95 \% \mathrm{Cl}=0.88-1.45$

Survival did not vary by place of residence (Cox proportional hazards regression analyses not presented)

Cox proportional hazards model $(\mathrm{HR}, 95 \% \mathrm{Cl}, p)$ : major city $=1.00$; inner regional $=0.65(0.32-1.32$,

$0.235)$; outer regional $=1.50(0.78-2.90,0.225)$;

remote $=1.43(0.48-4.29,0.522)$
Not significant $(p>0.001) \quad$ High (16) No significant difference in survival rates

Not significant $(p>0.05) \quad$ High (14) between metropolitan and non-metropolitan areas. for metropolitan versus regional patients was noted for colorectal cancer.

No significant difference in survival for metastatic colorectal cancer across regions.
No significant difference in median survival
$95 \% \mathrm{Cls}$

High (17)

95\% Cl):

Major cities $=1.00$

Inner regional = 1.02, 0.99-1.05

Outer $\mathrm{regional} / \mathrm{remote}=1.12,1.08-1.17$

Colon cancer survival rates (SE):

urban $=54.0 \%( \pm 0.9)$; rural $=49.3 \%( \pm 1.6)$

Rectal cancer survival rates:

urban $=55.5 \%( \pm 1.2)$; rural $=50.4 \%( \pm 2.0)$
Considered statistically significant if standard errors did not overlap.
Moderate

(13)

\section{Key findings}

Tests of significance

Study quality

Compared with major cities, living in outer regional/ remote areas was generally associated with a slightly increased risk of cancer death for colorectal cancers.

Survival rates from both colon and rectal cancer were significantly higher in urban areas compared to rural areas, after adjusting for age and sex.

SMR: 1.10* inner regional, 1.10* outer regional, 0.86 in remote areas, and $0.64^{*}$ in very remote areas, compared to major cities.

Excess death: inner regional $=95$, outer regional $=43$, remote $=-7$, very remote $=-8$.

Survival based on area $(95 \% \mathrm{Cl})$ : major cities $=67.2 \%(66.5-67.8)$; inner regional $=64.9 \%(63.8-66.0)$; outer regional $=62.6 \%$ (60.9-64.3); remote $=62.8(58.3-67.1)$.

\section{${ }^{*}$ Considered significant as p High (15) Death rates in regional areas were $10 \%$ \\ $=<0.05$ \\ higher than in major centres. Death rates in remote areas were lower, but not signifi- cantly lower, than those in major cities.}

$95 \% \mathrm{Cls}$

High (14)
Survival significantly higher in major cities than in inner and outer regional areas.
ASR: Major cities = 15.8; Inner regional = 15.8; Outer regional $=16.3 ;$ Remote $=14.1 ;$ Very remote $=10.9$ RR (relative to all of Australia): Major cities $=0.98$; Inner regional =1.03; Outer regional $=1.06$; Remote $=0.93 ;$ Very remote $=0.73$

Colon cancer 5 -year RS ( $95 \% \mathrm{Cl}$ ): major city $=67.9$ (66.8-69.1); inner regional = 65.6 (63.7-67.6); outer regional $=62.6(59.1-65.6)$; remote/very remote $=65.1$ (51.0-77.6).

Rectal cancer 5-year RS: major city $=67.3$ (65.868.7); inner regional $=64.8(62.4-67.2)$; outer regional $=63.5(59.2-67.7)$; remote $/$ very remote $=72.1$ (54.2-85.9).
High (14) Compared with major cities, people living in remote and very remote areas have an increased risk of mortality for colorectal cancers.

95\% Cls $\quad$ High (15)

Based on $95 \% \mathrm{Cl}$ the current authors note significantly lower survival rate for outer regional compared to major city for colon cancer and no significant differences for rectal cancer. 
TABLE 1 (Continued)

\begin{tabular}{|c|c|c|c|c|c|}
\hline Study & Design and data source & $\begin{array}{l}\text { Diagnosis } \\
\text { period }\end{array}$ & Population & Rural/urban definition & Outcome(s) \\
\hline $\begin{array}{l}\text { Cramb et al. } \\
\text { (2011) }\end{array}$ & $\begin{array}{l}\text { Atlas of cancer in } \\
\text { Queensland-retrospec- } \\
\text { tive state-wide popula- } \\
\text { tion registry study }\end{array}$ & 1998-2007 & $\begin{array}{l}\text { All cancers } \\
\text { diagnosed in } \\
\text { Queensland }\end{array}$ & ARIA+ (4 categories) & $\begin{array}{l}\text { Smoothed relative excess risk } \\
\text { (RER) by sex within five years of } \\
\text { diagnosis }\end{array}$ \\
\hline PHIDU (2012) & $\begin{array}{l}\text { Atlas of cancer in South } \\
\text { Australia-retrospective } \\
\text { state-wide population } \\
\text { registry study }\end{array}$ & 1995-2008 & $\begin{array}{l}\text { Colorectal cancer } \\
\text { patients aged } \\
0-74 \text { years }\end{array}$ & ASGC (3 categories) & $\begin{array}{l}\text { Cause-specific } 5 \text { year relative } \\
\text { survival rates (\%), age-standard- } \\
\text { ised mortality rates (ASR) and } \\
\text { Rate Ratios }\end{array}$ \\
\hline
\end{tabular}

\begin{tabular}{|c|c|c|c|c|c|}
\hline $\begin{array}{l}\text { Queensland } \\
\text { Health (2016) }\end{array}$ & $\begin{array}{l}\text { Retrospective popula- } \\
\text { tion-wide cohort study } \\
\text { (Queensland Oncology } \\
\text { Repository) }\end{array}$ & 2012 & $\begin{array}{l}\text { All colorectal } \\
\text { cancer patients } \\
\text { diagnosed in } \\
2012(N=2,788)\end{array}$ & ASGC ( 4 categories) & $\begin{array}{l}2 \text { year crude survival from diagno- } \\
\text { sis and RRs calculated by current } \\
\text { authors }\end{array}$ \\
\hline $\begin{array}{l}\text { Threlfall } \\
\text { \&Thompson } \\
\text { (2015) }\end{array}$ & $\begin{array}{l}\text { Retrospective popula- } \\
\text { tion-wide registry study } \\
\text { (WA) }\end{array}$ & 2014 & $\begin{array}{l}\text { All new cases of } \\
\text { cancer recorded } \\
\text { in WA in } 2014\end{array}$ & $\begin{array}{l}\text { Two WA metropolitan Area } \\
\text { Health Service (North and } \\
\text { South Metro AHS) and } \\
\text { seven WA country health } \\
\text { service (Kimberley, Pilbara, } \\
\text { Midwest, Wheatbelt, } \\
\text { Goldfields, Great Southern } \\
\text { \& South West) }\end{array}$ & $\begin{array}{l}\text { Age-standardised 1- year mortality } \\
\text { rate by sex (ASR) }\end{array}$ \\
\hline $\begin{array}{l}\text { Thursfield, } \\
\text { Farrugia, } \\
\text { Karahalios, \& } \\
\text { Giles (2012) }\end{array}$ & $\begin{array}{l}\text { Retrospective popula- } \\
\text { tion-wide registry study } \\
\text { (Victoria) }\end{array}$ & 1986-2010 & $\begin{array}{l}\text { All persons with a } \\
\text { first diagnosis of } \\
\text { cancer in Victoria }\end{array}$ & $\begin{array}{l}\text { Metropolitan is defined as } \\
\text { the three metropolitan } \\
\text { Integrated Cancer Service } \\
\text { (ICS) regions-Southern, } \\
\text { Western \& Central and } \\
\text { North-Eastern. The rest } \\
\text { of Victoria includes the } \\
\text { five rural ICS regions- } \\
\text { Barwon, Grampians, } \\
\text { Loddon-Mallee, Hume and } \\
\text { Gippsland. }\end{array}$ & $\begin{array}{l}\text { Cause-specific } 5 \text { year relative } \\
\text { survival rates }(\%)\end{array}$ \\
\hline
\end{tabular}

Abbreviations: ARIA, Accessibility/Remoteness Index of Australia; ASGC, Australian Standard Geographical Classification; NSW, New South Wales; QLD, Queensland; SA, South Australia; WA, Western Australia.

*Included in both Table 1 (survival differences) and Table 2 (stage at diagnosis)

\subsection{Measures of survival}

The reviewed studies report survival outcomes using a variety of different values including odds ratios (OR), hazard ratios (HR), standard mortality ratios (SMR) and relative excess risk ratios (RER). Subtle differences exist in the calculation of each ratio (see Table 3); however, their interpretation is analogous. That is, values lower than 1 represent a lower chance of an event occurring (i.e. death or survival), while values higher than 1 represent a higher chance of the event occurring.

\subsection{1 | Survival findings}

Of the 23 studies that investigated CRC survival disparities, 16 (69\%) reported evidence that living outside of a major city in Australia is 


\begin{tabular}{|c|c|c|c|}
\hline Key findings & Tests of significance & Study quality & Evidence summary \\
\hline $\begin{array}{l}\text { Survival rates }(95 \% \mathrm{Cl}) \text { for } 1995-2008 \text { : } \\
\text { Adelaide }=64.6(-62.6-63.7) \text {; inner regions }=66.2 \\
(-68.6-63.7) ; \text { more remote }=61.1(59.0-63.2) \\
\text { ASR 2003-2007: } \\
\text { Adelaide }=13.2 ; \text { non-metro } S A=10.5 \\
R R=1.27\end{array}$ & $95 \% \mathrm{Cls}$ & $\begin{array}{l}\text { Moderate } \\
\text { (13) }\end{array}$ & $\begin{array}{l}\text { Based on } 95 \% \mathrm{Cl} \text { the current authors } \\
\text { note that between } 1995 \text { and } 2008 \text { inner } \\
\text { regional and more remote residents had a } \\
\text { significantly higher risk of death that those } \\
\text { in Adelaide City. Recent data (2003-2007) } \\
\text { suggests that "premature" mortality rates } \\
\text { are higher in non-metro areas. }\end{array}$ \\
\hline $\begin{array}{l}\text { Males ASR }(95 \% \mathrm{CI}): \\
\text { country = } 8.7(6.0-11.4) \\
\text { metro }=11.6(9.9-13.3)\end{array}$ & $95 \% \mathrm{Cls}$ & $\begin{array}{l}\text { Moderate } \\
\text { (13) }\end{array}$ & $\begin{array}{l}\text { Based on } 95 \% \mathrm{Cl} \text { the current authors note no } \\
\text { significant differences between country and } \\
\text { metro survival rates. }\end{array}$ \\
\hline
\end{tabular}

country = $9.1(6.4-11.8)$

metro $=7.1(5.8-8.4)$

5-year survival rate $(95 \% \mathrm{Cl})$ :

$p<0.01 \quad$ High (16)

CRC survival was significantly higher in residents of Melbourne than those from the rest of the state of Victoria

associated with lower survival following CRC. Of the 12 studies that reported a cause-specific five-year survival analysis, 11 (91\%) reported significantly poorer survival outside of metropolitan areas, and four of five studies (80\%) that reported standardised mortality rates also reported worse survival in non-metropolitan areas. Four studies reported on relative risk and found increased risk of death from CRC with increasing remoteness. Five reviewed studies did not find significant geographical differences; two studies that reported on median survival length, one study that covered a shorter survival timeframe and was not age-adjusted, and two studies that reported on all-cause mortality. The majority of studies reviewed controlled for factors that have been found to impact survival including age and stage; however, this did not appear to effect the significance of findings. 
TAB LE 2 Characteristics of included studies (geographic variations in CRC stage at diagnosis)

\begin{tabular}{|c|c|c|c|c|c|}
\hline Study & Design & $\begin{array}{l}\text { Diagnosis } \\
\text { period }\end{array}$ & Population & Rural/urban definition & Outcome(s) \\
\hline \multicolumn{6}{|c|}{ Peer-reviewed studies } \\
\hline $\begin{array}{l}\text { Baade et al. } \\
\text { (2011b) }\end{array}$ & $\begin{array}{l}\text { Retrospective population- } \\
\text { wide registry study (QLD) }\end{array}$ & 1996-2007 & $\begin{array}{l}\text { Patients aged } \\
20-79 \text { years diagnosed } \\
\text { with invasive stage I-IV } \\
\text { CRC }(N=18,561)\end{array}$ & ARIA + (4 categories) & $\begin{array}{l}\text { Dichotomous outcome: "localised" } \\
\text { versus "regional and distal" }\end{array}$ \\
\hline $\begin{array}{l}\text { Beckmann et } \\
\text { al. }(2016)^{*}\end{array}$ & $\begin{array}{l}\text { Retrospective population- } \\
\text { wide registry study (SA) }\end{array}$ & 2003-2008 & $\begin{array}{l}\text { Cases of CRC aged } \\
50-79 \text { years }(N=4,362)\end{array}$ & ARIA (5 categories) & $\begin{array}{l}\text { Stage at diagnosis (ordinal outcome, } \\
\text { A to D) }\end{array}$ \\
\hline
\end{tabular}

\begin{tabular}{|c|c|c|c|c|c|}
\hline $\begin{array}{l}\text { Depczynski et } \\
\text { al. (2018) }\end{array}$ & $\begin{array}{l}\text { Retrospective population- } \\
\text { wide data linkage study } \\
\text { (NSW cancer registry and } \\
45 \text { and Up Study data) }\end{array}$ & 2006-2009 & $\begin{array}{l}\text { Patients diagnosed with } \\
\text { any cancer }(N=890 \\
\text { CRC cases) }\end{array}$ & $\begin{array}{l}\text { "Farm residents" were } \\
\text { defined as those who } \\
\text { indicated that they } \\
\text { lived in a "house on } \\
\text { farm" } \\
\text { Rural "non-farm" and } \\
\text { urban residents were } \\
\text { defined using ARIA+ (2 } \\
\text { categories) }\end{array}$ & $\begin{array}{l}\text { Degree of spread: (dichotomous out- } \\
\text { come Stage 1, "early stage" versus } \\
\text { Stage } 2 \text { or above "later stage" }\end{array}$ \\
\hline $\begin{array}{l}\text { Jong et al., } \\
2004^{*}\end{array}$ & $\begin{array}{l}\text { Retrospective population- } \\
\text { based cohort using NSW } \\
\text { cancer registry }\end{array}$ & 1992-1996 & $\begin{array}{l}\text { Patients diagnosed } \\
\text { with any cancer } \\
(N=132,516)\end{array}$ & ARIA (3 categories) & $\begin{array}{l}\text { Spread of cancer at diagnosis (or- } \\
\text { dinal outcome, localised, regional, } \\
\text { distant) }\end{array}$ \\
\hline
\end{tabular}

\begin{tabular}{|c|c|c|c|c|c|}
\hline Study & Design and data source & $\begin{array}{l}\text { Diagnosis } \\
\text { Period }\end{array}$ & Population & Rural/urban definition & Outcome(s) \\
\hline \multicolumn{6}{|c|}{ Grey Literature reports } \\
\hline $\begin{array}{l}\text { Armstrong et } \\
\text { al. (2004) }\end{array}$ & $\begin{array}{l}\text { Population-based cohort } \\
\text { using NSW colorectal } \\
\text { cancer care survey }\end{array}$ & 2000 & $\begin{array}{l}\text { CRC patients } \\
(N=3,095)\end{array}$ & ARIA (3 categories) & $\begin{array}{l}\text { Stage at diagnosis: Localised (Submu } \\
\text { Regional, and Distal }\end{array}$ \\
\hline
\end{tabular}

The pattern of results was not consistent across States and Territories or across studies applying different geographical classifications. All four studies from New South Wales showed significant geographical disparity in survival rates with poorer survival in nonmetropolitan areas; however, these findings differed when colon and rectal cancer were analysed separately (Cancer Institute NSW, 2018; Chen et al., 2015; Jong et al., 2004; Tervonen et al., 2017). Four of five studies in Queensland populations showed poorer survival with increasing distance from a major city (Baade, Dasgupta, Aitken, \& Turrell, 2011a, 2013; Cramb, Mengersen, \& Baade, 2011; Cramb, Mengersen, Turrell, \& Baade, 2012); however, none of the studies conducted in Western Australia $(N=3)$ found significant geographic disparity (Hall et al., 2005; Martin et al., 2015; Threlfall \& Thompson, 2015), and mixed results were evident in South Australian samples (Beckmann et al., 2016; Hocking et al., 2014; PHIDU, 2012; Roder et al., 2015; Singla, Broadbridge, Mittinty, Beeke, \& Maddern, 2014; Wilkinson \& Cameron, 2004) and using national data (Australian Institute of Health \& Welfare, 2007, 2012, 2017b; Coory, Ho, \&
Jordan, 2013). Fifteen studies utilised standard geographical classification structures such as the ASGC and the ARIA, using between two and five categories. Twelve of these (80\%) reported disparities in CRC survival across geographic locations, however, for eight of these studies geographic variations were not linear in nature (i.e. did not exhibit poorer survival as a linear function of increasing rurality). For instance, there was a trend for the lowest survival rates to be observed for inner and outer regional areas rather than remote or very remote areas.

The majority of included studies utilised data from approximately 10 or more years ago $(N=19 / 23,82 \%)$, with 13 of those studies reporting significantly poorer survival rates in non-metropolitan locations. Of the studies utilising newer data ( $<10$ years old), one using national data and one using data from New South Wales reported significant disparities in survival, while two state-specific studies from South Australia and Western Australia reported no significant geographic variations. This may indicate that geographical trends in CRC survival are changing over time. 


\begin{tabular}{|c|c|c|c|}
\hline Key findings & $\begin{array}{l}\text { Statistical inference of } \\
\text { differences }\end{array}$ & $\begin{array}{l}\text { Study } \\
\text { quality }\end{array}$ & Evidence summary \\
\hline $\begin{array}{l}\text { Probability of experiencing advanced stage CRC } \\
\text { (Odds Ratio, } 95 \% \mathrm{Cl}) \text { : major city }=1.00 \text { (ref); inner } \\
\text { regional }=1.09(1.01-1.19) \text {; outer regional }=1.11 \\
(1.01-1.22) ; \text { remote/very remote }=1.00 \\
(0.85-1.16) \\
\text { Analyses stratified by cancer site showed that } \\
\text { remoteness was significant for colon cancer } \\
(p=0.048) \text { but not for rectal cancer }(p=0.873)\end{array}$ & $\begin{array}{l}\text { Significance tested using } \\
\text { Z test: } \\
P \text {-value }=0.045\end{array}$ & High (17) & $\begin{array}{l}\text { Individuals from inner regional and outer regional } \\
\text { areas had significantly higher rates of advanced } \\
\text { CRC compared to major cities, while remote areas } \\
\text { had similar rates, after adjusting for sex, age, year of } \\
\text { diagnosis, Indigenous status, occupation, cancer site, } \\
\text { and marital status. }\end{array}$ \\
\hline $\begin{array}{l}\text { Adjusted odds ratio for factors associated with later } \\
\text { stage at diagnosis }(\mathrm{OR}, 95 \% \mathrm{Cl}) \text { : Inner urban }=1.00 \\
\text { (ref); outer urban }=0.89(0.74-1.07) \text {; rural }=0.99 \\
(0.83-1.16) \text {; remote }=0.99(0.77-1.26)\end{array}$ & $\begin{array}{l}\text { P values derived from } \\
\text { ordinal logistic regression } \\
(\text { all } p>0.05)\end{array}$ & High (16) & $\begin{array}{l}\text { Distribution of stage at diagnosis did not differ by } \\
\text { remoteness of residence in unadjusted analyses. } \\
\text { Multivariate analysis showed that stage at diagnosis } \\
\text { was not associated with place of residence. }\end{array}$ \\
\hline $\begin{array}{l}\text { Adjusted odds ratio for stage } 2+\text { CRC in men } \\
(95 \% \mathrm{CI}) \text { : Farm }=1.00 \text { ref, Rural non-farm }=0.45 \\
(0.19-1.07) \text {, urban }=0.49(0.21-1.17) \\
\text { Adjusted odds ratio for stage } 2+\mathrm{CRC} \text { in women } \\
(95 \% \mathrm{Cl}) \text { : Farm }=1.00 \text { ref, Rural non-farm }=1.21 \\
(0.47-3.09) \text {, urban }=0.92(0.36-2.35)\end{array}$ & $\begin{array}{l}\text { P values derived from } \\
\text { ordinal logistic regression } \\
(\text { all } p>0.25)\end{array}$ & High (14) & $\begin{array}{l}\text { Differences in stage of cancer diagnosis between rural } \\
\text { and urban were not statistically significant; however, } \\
\text { farm men had twice the odds of either non-farm rural } \\
\text { or urban men of being diagnosed at later stage CRC. }\end{array}$ \\
\hline $\begin{array}{l}\text { Ordinal logistic regression adjusting for age and } \\
\text { sex to compare spread of cancer between highly } \\
\text { accessible areas and the remaining areas. } \\
\text { Results not presented. }\end{array}$ & $p>0.05$ & High (17) & No significant difference in spread of CRC at diagnosis. \\
\hline Key findings & Tests of significance & $\begin{array}{l}\text { Study } \\
\text { quality }\end{array}$ & Evidence summary \\
\hline $\begin{array}{l}\text { Mean stage at diagnosis }(S D) \text { : } \\
\text { highly accessible }(M=2.33, S D=1.07) \text {; accessible } \\
(M=2.31, S D=1.04) \text {; moderately accessible/ } \\
\text { remote }(M=2.31, S D=1.13) \text {. } \\
\text { Remoteness did not predict advanced stage at } \\
\text { diagnosis }(O R=0.91,29 \% \mathrm{Cl}=0.73-1.14)\end{array}$ & $\begin{array}{l}\text { No statistical comparison } \\
\text { undertaken, current au- } \\
\text { thors evaluated } 95 \% \mathrm{Cls}\end{array}$ & $\begin{array}{l}\text { Moderate } \\
\text { (11) }\end{array}$ & $\begin{array}{l}\text { Based on tabled frequency data provided, the current } \\
\text { authors found no significant differences between } \\
\text { mean stage of diagnosis across regions. Highest } \\
\text { percentage of advanced cancer in remote areas was } \\
\text { not significant. }\end{array}$ \\
\hline
\end{tabular}

\section{5 | Cancer staging}

Cancer stage refers to the extent of disease at diagnosis, defined according to established criteria. For this review, CRC stage at diagnosis is considered within four categories: Stage I (localised), where the cancer is limited to the site of origin; Stage II (regional), where the cancer has spread beyond the initial site, often to the nearest lymph nodes; Stage III (distal), where the cancer has spread more extensively; and Stage $\mathrm{IV}$, where the cancer has spread to distant organs (metastatic disease).

\subsection{1 | Stage at diagnosis findings}

Only one of five reviewed studies reported disparities in stage at diagnosis, with individuals from regional areas showing significantly higher rates of advanced CRC compared to major cities, while remote areas had similar rates. However, these findings applied to colon cancer only and pertained to a Queensland only sample from 1996-2007 (Baade et al., 2011b).

\section{4 | DISCUSSION}

From the studies reviewed, there is strong and consistent evidence that in Australia, non-metropolitan CRC patients have significantly lower five-year survival compared to patients living in metropolitan areas. Insufficient evidence was available to draw meaningful conclusions about geographic disparities in stage at diagnosis for CRC patients. There are several lessons to be learned from the available data that warrant particular attention in understanding geographical variations, and in informing the direction and priorities for future research. In particular, these data provide important information about the current state of research in the area and improvements that can be made, with relevance to both Australia and internationally.

Findings from research conducted outside of Australia suggest that geographic disparities in survival may be explained by demographic factors such as ethnicity and socioeconomic status (Feller et al., 2018; Henry, Niu, \& Boscoe, 2009; Launoy, Le Coutour, Gignoux, Pottier, \& Dugleux, 1992; Singh, Williams, Siahpush, \& Mulhollen, 


\begin{tabular}{|c|c|}
\hline Ratio value & Interpretation \\
\hline Odds ratio (OR) & $\begin{array}{l}\text { ORs represent the chance of an event occurring (usually death, } \\
\text { but can represent survival) based on various other factors. It } \\
\text { assumes all data to be recorded at the same time point. In the } \\
\text { current context an OR is calculated as follows: } \\
\frac{D \div \text { Sforregional }}{D \div \text { Sformetropolitan }}\end{array}$ \\
\hline $\begin{array}{l}\text { Standardised mortality } \\
\text { ratio (SMR) }\end{array}$ & $\begin{array}{l}\text { SMRs represent the ratio of observed deaths in the study group } \\
\text { to expected deaths in the general population. Similar to the } \\
\text { calculation for ORs, SMRs are calculated as follows: } \\
\frac{D \div \text { Sforregional }}{D \div \text { Sforgeneralpopulation }}\end{array}$ \\
\hline Hazard ratio (HR) & $\begin{array}{l}\text { HRs represent and OR at a given point in time. That is, an HR } \\
\text { value is the times an event occurs more frequently per unit of } \\
\text { time (e.g. } 5 \text { years since diagnosis) }\end{array}$ \\
\hline $\begin{array}{l}\text { Relative excess risk (RER } \\
\text { or ERR) }\end{array}$ & $\begin{array}{l}\text { RERs is the difference between two relative risk ratios (RR) } \\
\text { represent the excess risk of an event occurring between two } \\
\text { groups compared to a baseline } \\
\left(\frac{D \div D / \text { forregional }}{D \div D / \text { Sformetropolitan }}\right)-\left(\frac{D \div D / \text { Sforregional }}{D \div D / \text { fformetropolitan }}\right)\end{array}$ \\
\hline
\end{tabular}

TAB LE 3 Ratio values and their interpretations in the context of cancer survival in a regional versus metropolitan comparison

Abbreviations: $D=$ death, $S=$ survival.

2012). In addition, several other factors may be at play including quality of rural services accessed, the availability of private facilities, or differences in emergency admissions that contribute to geographical disparity in CRC survival rather than remoteness itself (Baade et al., 2011b; Wan, Zhan, Lu, \& Tiefenbacher, 2012). For instance, surgeon expertise and hospital volume both influence CRC outcomes (Beckmann, Moore, Wattchow, Young, \& Roder, 2016; Harmon et al., 1999; Hodgson, Fuchs, \& Ayanian, 2001). Patients living in regional areas may access local, lower volume, public hospitals while patients who receive treatment in a metropolitan area have access to a variety of providers. A more thorough understanding of the main causes of geographic disparity, above and beyond location and including other demographic contributors, will aid future service delivery models and procedures.

The National Bowel Cancer Screening Program (NBCSP) was introduced in Australia in 2006, with full implementation of the programme expected in 2020. All reviewed studies investigating stage at diagnosis were conducted well before this implementation. Thus, the majority of participants sampled in this review would not have been exposed to population-wide screening. The majority of these studies reported that stage at diagnosis did not differ across geographic locations (Armstrong, O'Connell, Leong, Spigelman, \& Armstrong, 2004; Baade et al., 2011b; Beckmann et al., 2016; Depczynski, Dobbins, Armstrong, \& Lower, 2018; Jong et al., 2004). Based on this, suggestions that late diagnosis may explain disparities in CRC survival and outcomes are not strongly supported. It may be that geographic disparity in stage of CRC diagnosis itself does not exist, or alternatively that the current evidence does not allow us to reach this conclusion. Many countries worldwide have adopted population-level CRC screening (Swan, Siddiqui, \& Myers, 2012), with findings suggesting that early detection through screening can reduce mortality (Hewitson, Glasziou, Watson, Towler, \& Irwig, 2008). However, there are insufficient data to determine whether screening has an effect on subsequent stage at diagnosis differentially across various geographic locations; largely because of different practices in collecting and defining stage data in international cancer registries (Walters et al., 2013). Future research needs to focus on investigating other factors that might lead to regional disparity in survival, as well as monitor geographic disparities in stage at diagnosis with population-level data collected from the beginning of screening programmes onwards. It is important that all data relating to stage at diagnosis and survival of CRC are collected, analysed and reported in a standardised manner, in order to produce statistics that are comparable across time points.

Several studies analysed survival and stage at diagnosis separately for colon and rectal cancer, often reporting significant findings only for one cancer type. This suggests that studies analysing data on CRC as a whole may miss important differences. This is supported by research outside of Australia which suggests that differences in survival and stage at diagnosis in rural areas may differ according to location of the tumour (Feller et al., 2018; Hines \& Markossian, 2012). As Australian National Health and Medical Research Council guidelines for the clinical management of CRC provide different recommendations for colon and rectal cancer, access to chemotherapy services or radiotherapy facilities may have a differential impact on patients living in rural areas depending on their diagnosis. The reviewed studies do not provide clinical information on treatment, thus caution is warranted in interpreting the current findings. Access to screening and appropriate treatments may be an important indicator of survival from CRC and thus, upto-date comprehensive data are required to fully understand geographic disparities in CRC survival and stage at diagnosis.

Reviewed studies came from several different Australian states, often with varied findings. Each state has a different population distribution, climate and health system, making comparisons between states difficult. For instance, compared with other Australian states, Queensland has one of the highest proportions of Indigenous residents, and the highest decentralised population 
on the mainland with $52 \%$ of the population living outside of the capital city (Australian Bureau Statistics, 2016; Australian Institute of Health \& Welfare, 2015). International research suggests that advanced cancer is more likely to be diagnosed among Indigenous populations compared with Caucasian, and among persons living in low socioeconomic areas (Condon, Barnes, Armstrong, SelvaNayagam, \& Elwood, 2005; Henry et al., 2009; Tervonen et al., 2016). In addition, studies in the United States (McLafferty \& Wang, 2009; Parikh-Patel, Bates, \& Campleman, 2006), Scotland (Campbell et al., 2001) and Switzerland (Feller et al., 2018) have reported that geographic variability in stage at diagnosis of CRC may reflect the different population compositions in regional areas such as lower socioeconomic and larger indigenous populations, as well as access and availability of health services. Thus, there is a complex relationship between the factors that make rural and regional areas unique and potentially influence cancer survival, and which has yet to be thoroughly explored.

\subsection{Strengths and limitations}

Although the current review shares some overlap with a previous systematic review (Ireland et al., 2017), it captures data from an additional five government reports, two state non-profit cancer organisation reports, and two peer-reviewed papers published since 2017. These nine additional documents make up $35 \%$ of the sample of the current review. Given the rich data collected by state and national government agencies relating to survival and stage of diagnosis, the current review provides a more cohesive picture of this specific sub-topic, thus providing an additional contribution to the literature.

It is challenging to adequately compare the available evidence as several different methods were employed. For example, some authors report 1- or 2-year survival rates, which is less informative than 5-year survival, as patients may still be undergoing treatment and are not considered in remission (Parkin, Bray, Ferlay, \& Pisani, 2001). Other authors report length of survival, rather than mortality rates, which yields a different and non-comparable outcome measure. Second, the way in which each study classifies remoteness status varied in important ways. Some studies simply compared non-metropolitan to metropolitan and others represented remoteness as a continuous "distance" variable. Considering that the effect of remoteness on survival or stage at diagnosis may not be entirely linear, these methods are problematic and may further confound our understanding of the true patterns of disparity.

\subsection{Recommendations}

In order to determine the nature of geographic disparities in CRC survival and stage at diagnosis, population-level studies need to be replicated and standardised. It is recommended that future research examine current data from each state of Australia separately using a consistent and robust method of defining and reporting survival and classifying geographical location. The use of 5-year survival analyses and consistent categorisation of remoteness, preferably using a standard 3+ category system such as the ARIA would assist. Considering the implementation of the National Bowel Cancer Screening Program in 2006, it is recommended that CRC survival and stage of diagnosis be re-addressed in a contemporary sample. Again, state-by-state comparisons and comparable categorisation of key variables are paramount in understanding if and where disparity exists, and where intervention is required. Research aimed at improving cancer outcomes and detection in regional areas should continue, however, more recent data are required to maintain an upto-date understanding of disparities.

\section{5 | CONCLUSIONS}

The purpose of this review was to provide an outline of geographic disparities in CRC survival and stage at diagnosis, to provide a foundation and reference point from which to examine any change that might occur following the introduction and roll-out of the NBCSP. There is high-quality evidence to suggest poorer five-year survival outside of metropolitan areas in Australia, but this is influenced by stage and type of cancer, specific geographic regions, and the time period from which the data were collected. This early evidence suggests geographic variations in survival of CRC exists which has an important impact on policy and services. However, the impact of population-level screening needs to be further explored, and the issue of non-standardised reporting needs to be addressed. In general, while survival disparities are apparent, there does not appear to be a simple linear trend of disadvantage increasing with distance from metropolitan centres. This implies that geographic disparity is not simply related to remoteness, but likely linked to a range of individual, health system and diseasespecific factors. Further research would be beneficial to ascertain to what extent differences in cancer screening and treatment options affect survival for individuals diagnosed with CRC, so that healthcare services can be improved and interventions and policy changes can be guided by appropriate evidence. If data continues to be reported in an unstandardised manner, this will limit our ability to detect any impact of population-level CRC screening on stage at diagnosis and survival across differing geographic locations. As we reach full implementation of population-wide screening in Australia it is important that we have an understanding of the current state of the survival and stage disparities in order to track progress moving forward. This can only be done if current data are routinely and reliably collected and reported.

\section{ORCID}

Fiona Crawford-Williams (iD https://orcid. org/0000-0002-3897-333X

Belinda C. Goodwin (iD https://orcid.org/0000-0002-3425-4848

Suzanne K. Chambers iD https://orcid.org/0000-0003-2369-6111 


\section{REFERENCES}

Armstrong, K., O'Connell, D., Leong, D., Spigelman, A., \& Armstrong, B. K. (2004). The New South Wales Colorectal Cancer Care Survey 2000 Part 1 surgical management. Kings Cross, NSW: The Cancer Council NSW.

Australian Bureau Statistics (2016). Australian demographic statistics, Jun 2015. Canberra, ACT, Australia: ABS.

Australian Institute of Health and Welfare (2007). Rural, regional and remote health: A study on mortality. Canberra, ACT, Australia: AlHW.

Australian Institute of Health and Welfare (2012). Cancer survival and prevalence in Australia: Period estimates from 1982 to 2010 (Cancer Series). Canberra, ACT, Australia: AIHW.

Australian Institute of Health and Welfare (2015). The health and welfare of Australia's Aboriginal and Torres Strait Islander peoples: 2015 (IHW). Canberra, ACT, Australia: AlHW.

Australian Institute of Health and Welfare (2016). National bowel cancer screening program: Monitoring report 2016. Canberra, ACT, Australia: AlHW.

Australian Institute of Health and Welfare (2017a). Burden of cancer in Australia: Australian burden of disease study 2011 (Australian Burden of Disease Study). Canberra, ACT, Australia: AlHW.

Australian Institute of Health and Welfare (2017b). Cancer in Australia 2017 (Cancer series). Canberra, ACT, Australia: AIHW.

Baade, P. D., Dasgupta, P., Aitken, J. F., \& Turrell, G. (2011a). Distance to the closest radiotherapy facility and survival after a diagnosis of rectal cancer in Queensland. Medical Journal of Australia, 195(6), 350-354.

Baade, P. D., Dasgupta, P., Aitken, J. F., \& Turrell, G. (2011b). Geographic remoteness and risk of advanced colorectal cancer at diagnosis in Queensland: A multilevel study. British Journal of Cancer, 105(7), 1039-1041.

Baade, P. D., Dasgupta, P., Aitken, J. F., \& Turrell, G. (2013). Geographic remoteness, area-level socioeconomic disadvantage and inequalities in colorectal cancer survival in Queensland: A multilevel analysis. BMC Cancer, 13, 493. https://doi.org/10.1186/1471-2407-13-493

Beckmann, K., Bennett, A., Young, G., Ackland, S., Joshi, R., Adams, J., ... Roder, D. (2016). Sociodemographic disparities in survival from colorectal cancer in South Australia: A population-wide data linkage study. BMC Health Services Research, 16(1), 24. https://doi. org/10.1186/s12913-016-1263-3

Beckmann, K., Moore, J., Wattchow, D., Young, G., \& Roder, D. (2016). Short-term outcomes after surgical resection for colorectal cancer in South Australia. Journal of Evaluation in Clinical Practice, 23, 316-324. https://doi.org/10.1111/jep.12612

Campbell, N. C., Elliott, A. M., Sharp, L., Ritchie, L. D., Cassidy, J., \& Little, J. (2001). Rural and urban differences in stage at diagnosis of colorectal and lung cancers. British Journal of Cancer, 84(7), 910.

Cancer Institute NSW (2018). Cancer statistics NSW. Retrieved from https://www.cancer.nsw.gov.au/cancer-statistics-nsw\#/analysis/ survival/

Chen, T. Y., Morrell, S., Thomson, W., Baker, D. F., Walton, R., Aranda, S., \& Currow, D. C. (2015). Survival from breast, colon, lung, ovarian and rectal cancer by geographical remoteness in New South Wales, Australia, 2000-2008. Australian Journal Rural Health, 23(1), 49-56. https://doi.org/10.1111/ajr.12172

Condon, J. R., Barnes, T., Armstrong, B. K., Selva-Nayagam, S., \& Elwood, J. M. (2005). Stage at diagnosis and cancer survival for Indigenous Australians in the Northern Territory. Medical Journal of Australia, 182(6), 277-280.

Coory, M., Ho, T., \& Jordan, S. J. (2013). Australia is continuing to make progress against cancer, but the regional and remote disadvantage remains. The Medical Journal of Australia, 199(9), 605-608.

Cramb, S. M., Mengersen, K. L., \& Baade, P. D. (2011). Identification of area-level influences on regions of high cancer incidence in
Queensland, Australia: A classification tree approach. BMC Cancer, 11, 311-321.

Cramb, S. M., Mengersen, K. L., Turrell, G., \& Baade, P. D. (2012). Spatial inequalities in colorectal and breast cancer survival: Premature deaths and associated factors. Health \& Place, 18(6), 1412-1421.

Depczynski, J., Dobbins, T., Armstrong, B., \& Lower, T. (2018). Stage of diagnosis of prostate, breast and colorectal cancer in farm residents compared with other rural and urban residents in New South Wales. Australian Journal of Rural Health, 26(1), 56-62.

Duckett, S. \& Griffits, K. (2016). Perils of place: Identifying hotspots of health inequality. Melbourne, Vic: Grattan Institute.

Dunn, J., Ng, S. K., Breitbart, W., Aitken, J., Youl, P., Baade, P. D., \& Chambers, S. K. (2013). Health-related quality of life and life satisfaction in colorectal cancer survivors: Trajectories of adjustment. Health and Quality of Life Outcomes, 11(1), 46.

Feller, A., Schmidlin, K., Bordoni, A., Bouchardy, C., Bulliard, J., Camey, B., ... Zwahlen, M. (2018). Socioeconomic and demographic inequalities in stage at diagnosis and survival among colorectal cancer patients: Evidence from a Swiss population-based study. Cancer Medicine, 7(4), 1498-1510.

Ferlay, J., Soerjomataram, I., Ervik, M., Dikshit, R., Eser, S., Mathers, C., ... Bray, F. (2013). GLOBOCAN 2012: Cancer incidence and mortality worldwide. Lyon: International Agency for Research on Cancer.

Hall, S. E., Holman, C. D., Platell, C., Sheiner, H., Threlfall, T., \& Semmens, J. (2005). Colorectal cancer surgical care and survival: Do private health insurance, socioeconomic and locational status make a difference? ANZ Journal of Surgery, 75(11), 929-935. https://doi. org/10.1111/j.1445-2197.2005.03583.x

Harmon, J. W., Tang, D. G., Gordon, T. A., Bowman, H. M., Choti, M. A., Kaufman, H. S., ... Cameron, J. L. (1999). Hospital volume can serve as a surrogate for surgeon volume for achieving excellent outcomes in colorectal resection. Annals of Surgery, 230(3), 404-404.

Heathcote, K. E., \& Armstrong, B. K. (2007). Disparities in cancer outcomes in regional and rural Australia (Vol. 31, p. 70). Cancer Forum, 31(2), 20.

Henry, K. A., Niu, X., \& Boscoe, F. P. (2009). Geographic disparities in colorectal cancer survival. International Journal of Health Geographics, 8, 48-48. https://doi.org/10.1186/1476-072X-8-48

Hewitson, P., Glasziou, P., Watson, E., Towler, B., \& Irwig, L. (2008). Cochrane systematic review of colorectal cancer screening using the fecal occult blood test (hemoccult): An update. The American Journal of Gastroenterology, 103(6), 1541.

Hines, R. B., \& Markossian, T. W. (2012). Differences in late-stage diagnosis, treatment, and colorectal cancer-related death between rural and urban African Americans and whites in Georgia. The Journal of Rural Health, 28(3), 296-305. https://doi. org/10.1111/j.1748-0361.2011.00390.x

Hocking, C., Broadbridge, V. T., Karapetis, C., Beeke, C., Padbury, R., Maddern, G. J., ... Price, T. J. (2014). Equivalence of outcomes for rural and metropolitan patients with metastatic colorectal cancer in South Australia. Medical Journal of Australia, 201(8), 462-466.

Hodgson, D. C., Fuchs, C. S., \& Ayanian, J. Z. (2001). Impact of patient and provider characteristics on the treatment and outcomes of colorectal cancer. JNCI: Journal of the National Cancer Institute, 93(7), 501-515. https://doi.org/10.1093/jnci/93.7.501

Ireland, M. J., March, S., Crawford-Williams, F., Cassimatis, M., Aitken, J. F., Hyde, M. K., ... Dunn, J. (2017). A systematic review of geographical differences in management and outcomes for colorectal cancer in Australia. BMC Cancer, 17(1), 95. https://doi.org/10.1186/ s12885-017-3067-1

Jong, K. E., Smith, D. P., Yu, X. Q., O'Connell, D. L., Goldstein, D., \& Armstrong, B. K. (2004). Remoteness of residence and survival from cancer in New South Wales. The Medical Journal of Australia, 180(12), 618-622. 
Launoy, G., Le Coutour, X., Gignoux, M., Pottier, D., \& Dugleux, G. (1992). Influence of rural environment on diagnosis, treatment, and prognosis of colorectal cancer. Journal of Epidemiology and Community Health, 46(4), 365-367. https://doi.org/10.1136/jech.46.4.365

Lejeune, C., Sassi, F., Ellis, L., Godward, S., Mak, V., Day, M., \& Rachet, B. (2010). Socio-economic disparities in access to treatment and their impact on colorectal cancer survival. International Journal of Epidemiology, 39(3), 710-717.

Liberati, A., Altman, D. G., Tetzlaff, J., Mulrow, C., Gøtzsche, P. C., loannidis, J. P. A., ... Moher, D. (2009). The PRISMA statement for reporting systematic reviews and meta-analyses of studies that evaluate healthcare interventions: Explanation and elaboration. BMJ, 339, b2700. https://doi.org/10.1136/bmj.b2700

Martin, H. L., Ohara, K., Chin, W., Davidson, A., Bayliss, E., Redfern, A., \& Khattak, M. A. (2015). Cancer services in Western Australia: A comparison of regional outcomes with metropolitan Perth. Australian Journal Rural Health, 23(5), 302-308. https://doi.org/10.1111/ajr.12218

McLafferty, S., \& Wang, F. (2009). Rural reversal? Rural-urban disparities in late-stage cancer risk in Illinois. Cancer, 115(12), 2755-2764.

Nguyen-Pham, S., Leung, J., \& McLaughlin, D. (2014). Disparities in breast cancer stage at diagnosis in urban and rural adult women: A systematic review and meta-analysis. Annals of Epidemiology, 24(3), 228-235. https://doi.org/10.1016/j.annepidem.2013.12.002

Parikh-Patel, A., Bates, J. H., \& Campleman, S. (2006). Colorectal cancer stage at diagnosis by socioeconomic and urban/rural status in California, 1988-2000. Cancer, 107(S5), 1189-1195.

Parkin, D. M., Bray, F., Ferlay, J., \& Pisani, P. (2001). Estimating the world cancer burden: Globocan 2000. International Journal of Cancer, 94(2), 153-156.

PHIDU (2012). An atlas of cancer in South Australia. Adelaide, SA, Australia: Public Health Information Development Unit (PHIDU), The University of Adelaide.

Queensland Government (2016). Queensland Colorectal Cancer Audit 2016. Brisbane: Queensland Health.

Roder, D., Karapetis, C. S., Wattchow, D., Moore, J., Singhal, N., Joshi, R., ... Price, T. J. (2015). Colorectal cancer treatment and survival: The experience of major public hospitals in South Australia over three decades. Asian Pacific Journal of Cancer Prevention, 16(6), 2431-2440.

Singh, G. K., Williams, S. D., Siahpush, M., \& Mulhollen, A. (2012). Socioeconomic, rural-urban, and racial inequalities in US cancer mortality: Part I-All cancers and lung cancer and Part II-Colorectal, prostate, breast, and cervical cancers. Journal of Cancer Epidemiology, 2011, 1-27.

Singla, A., Broadbridge, V., Mittinty, M., Beeke, C., \& Maddern, G. J. (2014). Rural populations have equal surgical and survival outcomes in metastatic colorectal cancer. Australian Journal Rural Health, 22(5), 249-256.

Swan, H., Siddiqui, A. A., \& Myers, R. E. (2012). International colorectal cancer screening programs: Population contact strategies, testing methods and screening rates. Practical Gastroenterology, 36(8), 20-29.
Tervonen, H. E., Aranda, S., Roder, D., Walton, R., Baker, D., You, H., \& Currow, D. C. (2016). Differences in impact of Aboriginal and Torres Strait Islander status on cancer stage and survival by level of socioeconomic disadvantage and remoteness of residence: A populationbased cohort study in Australia. Cancer Epidemiology, 41, 132-138. https://doi.org/10.1016/j.canep.2016.02.006

Tervonen, H. E., Aranda, S., Roder, D., You, H., Walton, R., Morrell, S., ... Currow, D. C. (2017). Cancer survival disparities worsening by socioeconomic disadvantage over the last 3 decades in New South Wales, Australia. BMC Public Health, 17, 691. https://doi.org/10.1186/ s12889-017-4692-y

Threlfall, T., \& Thompson, J. (2015). Cancer incidence and mortality in Western Australia, 2014. Perth, WA: Department of Health.

Thursfield, V., Farrugia, H., Karahalios, E., Giles, G. (2012). Cancer Survival Victoria 2012: Estimates of survival 2006-2010 (and comparisons with earlier periods). Melbourne: Cancer Council Victoria.

Veitch, C., Crossland, L., Steeghs, M., Ho, Y. H., \& Hanks, H. (2008). Patients' experiences of colorectal cancer and oncology services in north Queensland. Australian Journal of Primary Health, 14(3), 93-100.

Walters, S., Maringe, C., Butler, J., Brierley, J. D., Rachet, B., \& Coleman, M. P. (2013). Comparability of stage data in cancer registries in six countries: Lessons from the International Cancer Benchmarking Partnership. International Journal of Cancer, 132(3), 676-685.

Wan, N., Zhan, F. B., Lu, Y., \& Tiefenbacher, J. P. (2012). Access to healthcare and disparities in colorectal cancer survival in Texas. Health \& Place, 18(2), 321-329. https://doi.org/10.1016/j.healt hplace.2011.10.007

Wells, G. A., Shea, B., O'Connell, D., Peterson, J. E. A., Welch, V., Losos, M., \& Tugwell, P. (2000). The Newcastle-Ottawa Scale (NOS) for assessing the quality of nonrandomised studies in meta-analyses. Ottawa: Ottawa Health Research Institute.

Wilkinson, D., \& Cameron, K. (2004). Cancer and cancer risk in South Australia: What evidence for a rural-urban health differential? Australian Journal of Rural Health, 12(2), 61-66.

Youl, P., Dasgupta, P., Youlden, D., Aitken, J., Garvey, G., Zorbas, H., ... Baade, P. (2016). A systematic review of inequalities in psychosocial outcomes for women with breast cancer according to residential location and Indigenous status in Australia. Psycho-Oncology, 25, 11571167. https://doi.org/10.1002/pon.4124

How to cite this article: Crawford-Williams F, March S, Goodwin BC, et al. Geographic variations in stage at diagnosis and survival for colorectal cancer in Australia: A systematic review. Eur J Cancer Care. 2019;28:e13072. https://doi.org/10.1111/ecc.13072 
APPENDIX A

\section{RECORD OF SEARCH SYNTAX FOR EACH DATABASE}

\section{PubMed search query}

\begin{tabular}{ll}
\hline Search & Query \\
\hline \#1 & $\begin{array}{c}\text { colorectal neoplasm[MeSH Terms] OR colorectal cancer[Title/Abstract] OR bowel cancer[Title/Abstract] OR colon cancer[Title/Abstract] OR } \\
\text { rectal cancer[Title/Abstract] }\end{array}$ \\
\#2 & geography[MeSH Terms] OR urban population[MeSH Terms] OR urban health[MeSH Terms] OR rural population[MeSH Terms] OR \\
rural health[MeSH Terms] OR health status disparities[MeSH Terms] OR healthcare disparities[MeSH Terms] OR health services \\
accessibility[MeSH Terms] OR geograph*[Title/Abstract] OR metropolitan[Title/Abstract] OR urban[Title/Abstract] OR rural[Title/Abstract] \\
OR remote[Title/Abstract] OR regional[Title/Abstract] \\
survival[MeSH Terms] OR mortality[MeSH Terms] OR survival rate[MeSH Terms] OR survival analysis[MeSH Terms] OR neoplasm \\
staging[MeSH Terms] OR survival*[Title/Abstract] OR mortality[Title/Abstract] OR stage[Title/Abstract] OR spread[Title/Abstract]
\end{tabular}

\section{CINAHL via EbscoHost}

\begin{tabular}{ll} 
Search & Query \\
$\# 1$ & MH "colorectal neoplasm" OR "bowel cancer" OR "colon cancer" OR "rectal cancer" OR "colorectal cancer" \\
$\# 2$ & $\begin{array}{l}\text { MH geography OR MH "urban population" OR MH "rural population" OR MH "Rural Health Services" OR MH "Healthcare Disparities" } \\
\text { OR MH "Health Status Disparities" OR geography OR rural OR metropolitan OR urban OR remote OR regional }\end{array}$ \\
\#3 & MH survival OR MH mortality OR MH "survival analysis" OR MH "neoplasm staging" OR survival OR mortality OR stage \\
\hline
\end{tabular}

\section{Psyclnfo via Ebscohost}

\begin{tabular}{ll} 
Search & Query \\
$\# 1$ & MH "colorectal neoplasm" OR "bowel cancer" OR "colon cancer" OR "rectal cancer" OR "colorectal cancer" \\
$\# 2$ & MH geography OR MH "urban population" OR MH "rural population" OR MH "Rural Health Services" OR MH "Healthcare Disparities" \\
OR MH "Health Status Disparities" OR geography OR rural OR metropolitan OR urban OR remote OR regional & MH survival OR MH mortality OR MH "survival analysis" OR MH "neoplasm staging" OR survival OR mortality OR stage \\
\hline
\end{tabular}

\section{Informit}

\begin{tabular}{ll} 
Search & Query \\
\hline$\# 1$ & (mh:colorectal neoplasm OR "colorectal cancer" OR "bowel cancer" OR "colon cancer" OR "rectal cancer") \\
$\# 2$ & $\begin{array}{c}\text { mh:geography OR mh:urban population OR mh:rural population OR mh:rural health OR mh:healthcare disparities OR mh:health status } \\
\text { disparities OR geography OR rural OR metropolitan OR remote OR urban OR regional }\end{array}$ \\
$\begin{array}{l}\text { mh:survival OR survival OR mh:mortality OR mortality OR mh:survival analysis OR "survival analysis" OR mh:neoplasm staging OR } \\
\text { stage }\end{array}$ \\
\hline
\end{tabular}

\section{Embase}

\begin{tabular}{ll} 
Search & Query \\
\hline$\# 1$ & $\begin{array}{c}\text { ('colorectal cancer'/exp OR colorectal cancer OR 'bowel cancer'/exp OR bowel cancer OR 'colorectal neoplasm'/exp OR colorectal } \\
\text { neoplasm OR 'colon cancer'/exp OR colon cancer OR 'rectal cancer'/exp OR rectal cancer) }\end{array}$ \\
\#2 & $\begin{array}{l}\text { (geography/exp OR geography OR metropolitan/exp OR metropolitan OR "urban population"/exp OR "urban population" OR "remote } \\
\text { population"/exp OR "remote population" OR 'rural population'/exp OR 'rural population' OR 'rural health care'/exp OR 'rural health } \\
\text { care' OR 'urban rural difference'/exp OR 'urban rural difference' OR 'health disparity'/exp OR 'health disparity' OR 'health care dis- } \\
\text { parity'/exp OR 'health care disparity' }\end{array}$ \\
& ('survival'/exp OR survival OR 'mortality'/exp or mortality) \\
\hline \#3
\end{tabular}

